\title{
Nuclear Spin-Lattice Relaxation and Knight Shift in Liquid In-Zn System
}

\author{
R. Bucklisch and D. Ploumbidis \\ Institut für Atom- und Festkörperphysik der Freien Universität Berlin
}

Z. Naturforsch. 36a, 1155-1157 (1981); received September 30, 1981

The nuclear spin-lattice relaxation time $T_{1}$ and the Knight shift $K$ of ${ }^{115} \mathrm{In}$ in the liquid In-Zn system have been measured over the temperature range $(450-900) \mathrm{K}$. The Korringa correction factor $K(\alpha)$ is found to be temperature independent. The electric quadrupolar contribution to the relaxation rate is concluded to be very small (about $6 \%$ of the measured relaxation rate).

\section{Introduction}

Measurements of the spin-lattice relaxation time $T_{1}$ and the Knight shift $K$ in liquid metals and alloys contribute to a deeper understanding of the internal motions and the electronic structure of these materials. There are various mechanisms which can contribute to $K$ and $T_{1}$.

In most nontransition metals the dominant mechanism which couples the nuclear magnetic moment with the magnetic fields due to the conduction electrons is the contact hyperfine interaction. In this case $K$ is given by

$$
K_{\mathrm{S}}=\frac{8 \pi}{3} \chi_{\mathrm{s}} \Omega\left\langle\left|\Psi_{\mathrm{s}}(0)\right|^{2}\right\rangle_{E_{\mathrm{F}}},
$$

where $\chi_{\mathrm{s}}$ is the spin susceptibility for the s-electrons, $\Omega$ is the atomic volume, and $\left\langle\left|\Psi_{\mathrm{S}}(0)\right|^{2}\right\rangle_{E_{F}}$ is the probability density of s-electrons at the nuclear site averaged over the Fermi surface [1].

Other contributions to $K$ such as the orbital $K_{\text {orb }}$ and core polarization $K_{\text {cp }}$ are much weaker than $K_{\mathrm{s}}$ in simple metals. In transition metals $K_{\text {orb }}$ or $K_{\text {cp }}$ can be the main part of $K$ and they can have a dominant temperature dependence in comparison to the other contributions [2].

The spin lattice relaxation rate $R_{1}=1 / T_{1}$ is the sum of two significant parts [3]

$$
R_{1}=R_{1 \mathrm{~m}}+R_{1 \mathrm{Q}} \text {. }
$$

$R_{1 \mathrm{~m}}$ is the magnetic relaxation induced by the interaction between the nuclear dipole moment and the time-dependent magnetic fields, and $R_{1 \mathrm{Q}}$ is the quadrupolar relaxation, induced by the coupling

Reprint requests to Ass. Prof. Dr. D. Ploumbidis, Freie Universität Berlin, Institut für Atom- und Festkörperphysik, Königin-Luise-Str. 28/30, D-1000 Berlin 33. between the nuclear electric quadrupole moment and the time dependent electric field gradients. Korringa [4] derived first the expression for $R_{1 \mathrm{~m}}$ :

$$
\begin{aligned}
R_{1 \mathrm{~m}}= & \frac{64}{9} \pi^{3} h^{3} \gamma_{\mathrm{e}}^{2} \gamma_{\mathrm{n}}^{2}\left\langle\left|\Psi_{\mathrm{s}}(0)\right|^{2}\right\rangle_{E_{\mathrm{P}}} \\
& \cdot N^{2}\left(E_{\mathrm{F}}\right) K_{\mathrm{B}} T,
\end{aligned}
$$

where $\gamma_{\mathrm{e}}$ is the electronic gyromagnetic ratio, $\gamma_{\mathrm{n}}$ is the nuclear gyromagnetic ratio, $\left\langle\left|\Psi_{\mathrm{s}}(0)\right|^{2}\right\rangle_{E_{\mathrm{F}}}$ is as in (1), $N\left(E_{\mathrm{F}}\right)$ is the density of states at the Fermi surface for s-electrons, $K_{\mathrm{B}}$ is the Boltzmann constant and $T$ is the temperature. $R_{1 \mathrm{~m}}$ is related to $K_{\mathrm{s}}$ of (1) by the Korringa relation [4]:

$$
R_{1 \mathrm{~m}}=T K_{\mathrm{s}}^{2} \frac{4 \pi K_{\mathrm{B}}}{h} \frac{\gamma_{\mathrm{n}}^{2}}{\gamma_{\mathrm{e}}^{2}} .
$$

This equation is derived in the frame of the independent-electron approximation. For the effects of electron correlation and exchange on the Knight shift and the relaxation time accounts a correction factor $K(\alpha)$ introduced in (4) [5]. In this case a modified Korringa relation can be applied:

$$
R_{1 \mathrm{~m}}=T K_{\mathrm{s}}^{2} \frac{4 \pi K_{\mathrm{B}}}{h} \frac{\gamma_{\mathrm{n}}^{2}}{\gamma_{\mathrm{e}}^{2}} K(\alpha)
$$

We consider now the electric quadrupole contribution $R_{1 Q}$ to the relaxation rate $R_{1}$. The main contribution to $R_{1 Q}$ in liquid metals is the thermal modulation of the charge distribution around a nucleus by diffusional and vibrational motion. A direct quadrupolar relaxation caused by the conduction electrons has been estimated to be a mechanism capable of providing less than $1 \%$ of the observed $R_{1 \mathrm{Q}}$ in some cases [6].

Now an important purpose of this work is to evaluate the contributions of the various mecha- 
nisms to $R_{1}$ and $K$ in the liquid In-Zn system. Much recent activity on NMR studies of the liquid Indium alloys has been centered on development and testing of physical models describing the electronic structure and other physical properties [7-9]. In this paper we report the first measurements of the Knight shift $K$ and the spin lattice relaxation time $T_{1}$ for the ${ }^{115} \mathrm{In}$ isotope in liquid $\mathrm{In}-\mathrm{Zn}$ for the temperature range $(450-900) \mathrm{K}$. In addition to the measurements, an evaluation of the various mechanisms responsible for the observed values of $K$ and $T_{1}$ is presented.

\section{Results}

Figure 1 shows the measured Knight shift of ${ }^{115} \mathrm{In}$ in liquid $\mathrm{In}-\mathrm{Zn}$ as a function of temperature. The choice of the concentration range in the vicinity of 50 at.- \% In is because of our earlier NMR studies in the In-Hg liquid system [7]. We found at 50 at. $\% \mathrm{Hg}$ an absolute maximum of the 115In-Knight shift there, which is not the case for the In-Zn system. Experimental details and preparation of the NMR samples are described elsewhere [8].

As Fig. 1 shows, for temperatures below the melting point $T_{\mathrm{m}}$ of the $\mathrm{In}-\mathrm{Zn}$ alloys, $K\left({ }^{115} \mathrm{In}\right)$ decreases rapidly with decreasing temperature and tends to values close to those of $K(115 \mathrm{In})$ in pure Indium. This can be explained, if one supposes that the In-Zn alloy for temperatures lower than the melting point consists of two systems. One system is solidified $\mathrm{Zn}$ or $\mathrm{Zn}$-In (in a solid $\mathrm{Zn}$-In no InNMR-signals can be easily observed, mainly be-

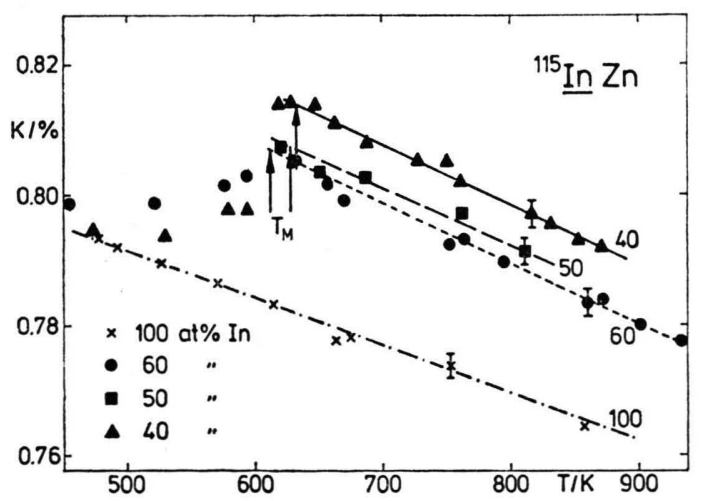

Fig. 1. The experimental Knight shift $K$ for ${ }^{115}$ In in liquid In- $\mathrm{Zn}$ system as a function of temperature for several concentrations. ( $T_{\mathrm{M}}$ : melting point).

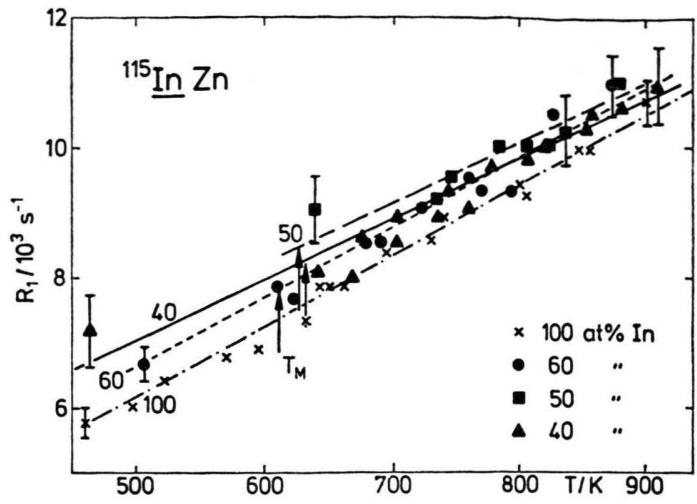

Fig. 2. The experimental relaxation rate $R_{1}=1 / T_{1}$ for 115In in liquid In-Zn system as a function of temperature for the same concentrations as for the Knight shift in Figure 1.

cause of quadrupolar line broadening) and the other is almost pure liquid Indium in which InNMR is detectable. Detailed measurements concerning Knight shift and relaxation time in the liquid In-Zn system as functions of concentration are subject of a further work.

Our experimental results for the spin-lattice relaxation rate $R_{1}=1 / T_{1}$ as a function of temperature for the ${ }^{115} \mathrm{In}-\mathrm{NMR}$ in the liquid In-Zn system, are plotted in Figure 2.

On the assumption that (5) describes adequately our system for $K\left({ }^{115} \mathrm{In}\right)$ and $T_{1}$ we calculated the

Table 1. The calculated values for the correction factor $K(\alpha)$ for different concentrations and temperatures for the liquid In-Zn system. The corresponding values of Knight shift $K\left({ }^{115} \mathrm{In}\right)$ and relaxation time $T_{1}$ are also given.

\begin{tabular}{|c|c|c|c|}
\hline$T(\mathrm{~K})$ & $K(\%)$ & $T_{1}(\mathrm{~ms})$ & $K(\alpha)$ \\
\hline \multicolumn{4}{|l|}{40 at. $\%$ In } \\
\hline $\begin{array}{l}650 \pm 0.4 \% \\
700 \pm 0.4 \% \\
800 \pm 0.4 \% \\
900 \pm 0.4 \%\end{array}$ & $\begin{array}{l}0.8124 \pm 0.3 \% \\
0.8078 \pm 0.3 \% \\
0.7985 \pm 0.3 \% \\
0.7892 \pm 0.3 \%\end{array}$ & $\begin{array}{l}0.119 \pm 5 \% \\
0.112 \pm 5 \% \\
0.102 \pm 5 \% \\
0.093 \pm 5 \%\end{array}$ & $\begin{array}{l}1.07 \pm 5 \% \\
1.07 \pm 5 \% \\
1.05 \pm 5 \% \\
1.05 \pm 5 \%\end{array}$ \\
\hline \multicolumn{4}{|l|}{50 at. $\%$ In } \\
\hline $\begin{array}{l}650 \pm 0.4 \% \\
700 \pm 0.4 \% \\
800 \pm 0.4 \% \\
850 \pm 0.4 \%\end{array}$ & $\begin{array}{l}0.8053 \pm 0.4 \% \\
0.8010 \pm 0.4 \% \\
0.7924 \pm 0.4 \% \\
0.7880 \pm 0.4 \%\end{array}$ & $\begin{array}{l}0.116 \pm 5 \% \\
0.110 \pm 5 \% \\
0.099 \pm 5 \% \\
0.095 \pm 5 \%\end{array}$ & $\begin{array}{l}1.12 \pm 5 \% \\
1.11 \pm 5 \% \\
1.10 \pm 5 \% \\
1.09 \pm 5 \%\end{array}$ \\
\hline \multicolumn{4}{|l|}{60 at. $\%$ In } \\
\hline $\begin{array}{l}650 \pm 0.4 \% \\
700 \pm 0.4 \% \\
800 \pm 0.4 \% \\
900 \pm 0.4 \%\end{array}$ & $\begin{array}{l}0.8034 \pm 0.4 \% \\
0.7987 \pm 0.4 \% \\
0.7894 \pm 0.4 \% \\
0.7801 \pm 0.4 \%\end{array}$ & $\begin{array}{l}0.122 \pm 5 \% \\
0.114 \pm 5 \% \\
0.102 \pm 5 \% \\
0.092 \pm 5 \%\end{array}$ & $\begin{array}{l}1.07 \pm 5 \% \\
1.08 \pm 5 \% \\
1.08 \pm 5 \% \\
1.09 \pm 5 \%\end{array}$ \\
\hline
\end{tabular}


correction factor $K(\alpha)$. The values of $K(\alpha)$ for several temperatures and concentrations are given in Table 1.

\section{Discussion}

As Table 1 shows $K(\alpha)$ is a constant within experimental error over the entire temperature range and the several concentrations. The Korringa relation predicts that the product $K_{\mathrm{s}}{ }^{2} T_{1} T$ is a constant (cf. (4)) and this is what we also find from our experimental results. We give for this product for the 50 at. $\%$ In -50 at. $\% \mathrm{Zn}$ system a representative value:

$$
K^{2} T_{1} T=4.95 \cdot 10^{-6}(\mathrm{sK}) .
$$

Now the constancy and the magnitude of $K(\alpha)$ indicate that the magnetic contact hyperfine interaction is the most dominant contribution to

[1] C. H. Townes, C. Herring, and W. D. Knight, Phys. Rev. 77, 852 (1950).

[2] D. Ploumbidis, Z. Phys. B 29, 61 (1977).

[3] T. E. Faber, Sol. St. Commun. 1, 41 (1963).

[4] J. Korringa, Physica 16, No. 7-8, 601 (1950).

[5] C. P. Slichter, Principles of Magnetic Resonance, Springer-Verlag Berlin 1980, p. 149.
$K\left({ }^{115} \mathrm{In}\right)$ and $T_{1}$ for the liquid $\mathrm{In}-\mathrm{Zn}$ system. It is expected that there is an electric quadrupolar contribution to $T_{1}$. From our experimental results and their interpretation in connection with the Korringa relation appears that the quadrupolar contribution to $R_{1}$ is negligibly small. We can make an estimate of the quadrupolar rate $R_{1 Q}$ assuming that (4) is valid for the liquid In-Zn system, that is we assume that $K(\alpha)=1$. With the measured values for $K\left({ }^{115} \mathrm{In}\right)$ and $T_{1}$ we get $R_{1 \mathrm{~m}}$ from (4). Equation (2) gives then with the experimentally determined $R_{1}=1 / T_{1}$ the value for $R_{1 Q}$. So we find that the quadrupolar relaxation rate $R_{1 Q}$ amounts to about $6 \%$ of the measured entire relaxation rate $R_{1}$.

\section{Acknowledgement}

This work was supported by the Deutsche Forschungsgemeinschaft.

[6] F. A. Rossini and W. D. Knight, Phys. Rev. 178, 641 (1969).

[7] D. Ploumbidis and T. Sutter, Phys. Stat. Sol. (b) 91, 185 (1979).

[8] D. Ploumbidis and R. Bucklisch (in preparation for publication).

[9] M. v. Hartrott, J. Höhne, D. Quitmann, J. Rossbach, E. Weihreter, and F. Willeke, Phys. Rev. B 19, 3449 (1979). 